\title{
Single-Electron Devices and Circuits in Silicon
}


This page intentionally left blank 


\section{Single-Electron Devices and Circuits in Silicon}

\section{Zahid Ali Khan Durrani}

Imperial College, UK 
Published by

Imperial College Press

57 Shelton Street

Covent Garden

London WC2H 9HE

\section{Distributed by}

World Scientific Publishing Co. Pte. Ltd.

5 Toh Tuck Link, Singapore 596224

USA office: 27 Warren Street, Suite 401-402, Hackensack, NJ 07601

UK office: 57 Shelton Street, Covent Garden, London WC2H 9HE

\section{British Library Cataloguing-in-Publication Data}

A catalogue record for this book is available from the British Library.

\section{SINGLE-ELECTRON DEVICES AND CIRCUITS IN SILICON}

Copyright () 2010 by Imperial College Press

All rights reserved. This book, or parts thereof, may not be reproduced in any form or by any means, electronic or mechanical, including photocopying, recording or any information storage and retrieval system now known or to be invented, without written permission from the Publisher.

For photocopying of material in this volume, please pay a copying fee through the Copyright Clearance Center, Inc., 222 Rosewood Drive, Danvers, MA 01923, USA. In this case permission to photocopy is not required from the publisher.

ISBN-13 978-1-84816-413-0

ISBN-10 1-84816-413-0

Printed in Singapore. 
To my wife, Olivia, and to my parents, Khalid and Rahila 
This page intentionally left blank 


\section{Preface}

Single-electron devices provide a means to control electronic charge at the level of one electron, by means of the single-electron charging or 'Coulomb blockade' effect. These devices operate by controlling the transfer of charge across tunnel barriers onto nanometre-scale conducting regions or 'islands'. In such a process, the energy needed to charge an island with even one electron can be large enough to influence the tunnelling process. This energy, the 'single-electron charging energy', must be overcome to allow current to flow across the island, preventing current flow at low applied voltage and temperature.

The possibility that the single-electron charging energy of a nanostructure could influence the tunnelling of even one electron onto the nanostructure was identified as early as the 1950s. In 1951, C.J. Gorter proposed that the observed increase in resistance in thin, granular metal films at low electric fields and temperatures was associated with the need to overcome the single-electron charging energy of the nanometre-scale grains in the film. In the mid-1980s, K.K. Likharev and co-workers predicted, in great detail, effects relating to single-electron charging in nanometre-scale tunnel junctions. By this stage, advances in nanofabrication techniques had led to the ability to fabricate well-defined, nanometre-scale, islands and tunnel junctions. In 1987, this led to the first demonstration, at low temperature, of a designed single-electron device, the single-electron transistor (SET) of Fulton and Dolan.

Over the following two decades, a wide variety of single-electron devices, in many material systems, were demonstrated. These included numerous different types of SETs, single-electron memory devices, single-electron logic circuits, and devices for the controlled transfer of 
charge packets formed by single or small numbers of electrons. Furthermore, there were many demonstrations of the room-temperature operation of single-electron devices, particularly in silicon semiconductor material.

The great interest in single-electron devices has been driven in part by the potential of these devices for applications in large-scale integrated (LSI) circuits. In comparison with conventional semiconductor devices, devices such as the SET and the single-electron memory cell are inherently nanometre-scale, and tend to improve in performance when scaled down in size. Furthermore, these devices possess the advantages of very low power consumption, associated with the small amounts of charge they use, and control over any statistical fluctuations in this charge.

This book discusses the design, fabrication and electrical characterization of single-electron devices and circuits in silicon. We concentrate on single-electron devices in silicon, as these are of particular interest for LSI circuit applications. Single-electron devices in metals, and in other semiconductor systems such as GaAs/AlGaAs heterostructures, are discussed only when necessary to understand the operation of particular device types, or when device implementations in silicon are limited. This book considers the physics of single-electron charging effects. This is followed by a review of the fabrication and operation of SETs in crystalline and nanocrystalline silicon materials. Single-electron memory devices are then discussed, where the stored 'bits' are defined by single, or at most a few, electrons. We then consider few-electron charge transfer devices, such as single-electron pumps and turnstiles, where small numbers of electrons can be transferred through the device using radio frequency signals. Finally, we discuss singleelectron logic circuits. Throughout this book, we follow an approach where the various types of single-electron devices are reviewed first, and then explained in more detail using examples from the author's research.

The author's research work would not have been possible without the collaboration and support of many colleagues. Firstly, the author would like to acknowledge the help and support of Prof. Haroon Ahmed at the Microelectronics Research Centre, University of Cambridge, UK. The major part of the author's research was conducted in collaboration with 
Prof. Ahmed, and would not have been possible otherwise. The author would also like to acknowledge the great support of, and collaborations with, many others at the Microelectronics Research Centre, especially Dr John Cleaver, Dr Andrew Irvine, Dr David Hasko, Dr Yong Tsong Tan, Dr Toshio Kamiya, Dr Mohammed Khalafalla, Dr Jin He and Dr Aftab Rafiq (now at Tokyo Institute of Technology, Japan). This research was also conducted in collaboration with Hitachi Cambridge Laboratory, and in this regard, the author would like to acknowledge extremely valuable collaborations with, and the help and support of, Prof. Hiroshi Mizuta (now Professor of Nanoelectronics at the University of Southampton, UK), Prof. Kazuo Nakazato (now at the University of Nagoya, Japan) and Dr David Williams. Furthermore, a large part of the author's research has been possible only through longstanding and very productive collaborations with Prof. Shunri Oda at Tokyo Institute of Technology, Japan, and with Prof. William Milne at the Department of Engineering, University of Cambridge, UK. The author would also like to acknowledge the help and support of Prof. Richard Syms, Prof. Mino Green and Dr Kristel Fobelets, at the Department of Electrical and Electronic Engineering, Imperial College, London, UK, and the support of many other colleagues and friends at the Microelectronics Research Centre, Cambridge, Hitachi Cambridge Laboratory and the Department of Engineering, University of Cambridge. The author would like to acknowledge the financial support of various research programmes. These include the FASEM project (part of the ESPRIT programme of the European Union), the 'Single-electron logic' project, funded by EPSRC, UK, and the CREST and SORST programmes of Japan Science and Technology Corp., Japan.

Finally, the author would like to acknowledge the invaluable support and encouragement of his wife, Olivia Kesselring-Durrani, his parents, Khalid and Rahila Durrani, and his mother-in-law, Ursula Kesselring. Their support has made the writing of this book a far less arduous task. 
This page intentionally left blank 


\section{Contents}

Preface vii

1. Introduction 1

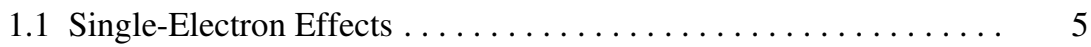

1.2 Early Observations of Single-Electron Effects . . . . . . . . . . 8

1.3 Basic Single-Electron Devices . . . . . . . . . . . . . . . 12

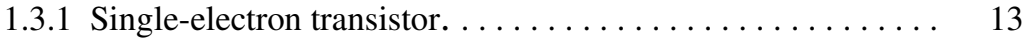

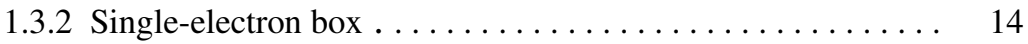

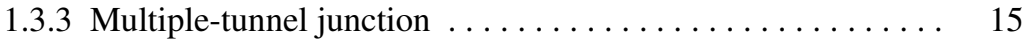

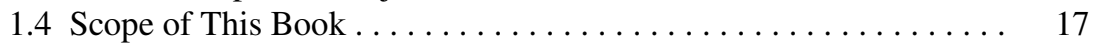

1.4.1 Introduction to subsequent chapters $\ldots \ldots \ldots \ldots \ldots$

2. Single-Electron Charging Effects 22

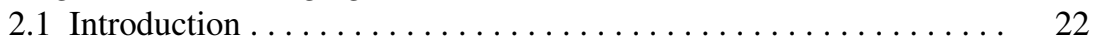

2.2 Single Tunnel Junction . . . . . . . . . . . . . . . . . . . 25

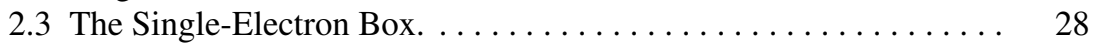

2.3.1 The 'critical charge'. . . . . . . . . . . . . . . . . . . 29

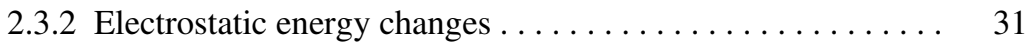

2.3.3 Energy diagram for the single-electron box. . . . . . . . 36

2.4 The Single-Electron Transistor . . . . . . . . . . . . . . 37

2.4.1 Electrostatic energy changes . . . . . . . . . . . . 39

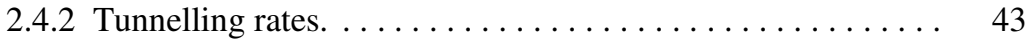

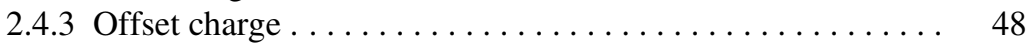

2.4.4 Calculation of $I-V$ characteristics. . . . . . . . . . . 49

2.4.5 The Coulomb staircase ................. 52

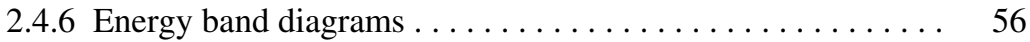

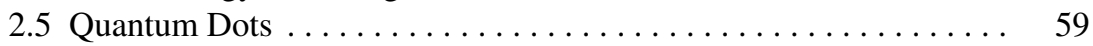

2.5.1 Coulomb oscillations in quantum dots. . . . . . . . . 62

2.6 The Multiple-Tunnel Junction . . . . . . . . . . . . . . . . . 64 
3. Single-Electron Transistors in Silicon 72

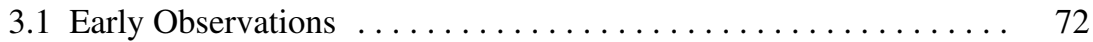

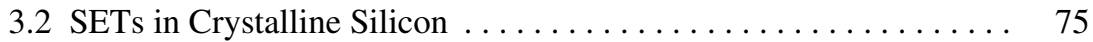

3.2.1 SETs with lithographically defined islands ......... 75

3.2.1.1 Etched islands. . . . . . . . . . . . . . 76

3.2.1.2 Pattern-dependant oxidation $\ldots \ldots \ldots \ldots \ldots \ldots .62$

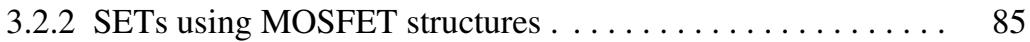

3.2.3 Crystalline silicon nanowire SETs ............. 87

3.2.4 Room temperature Coulomb oscillations with large peak-tovalley ratio ........................ 91

3.2.5 Fabrication and characterization of nanowire SETs . . . . . 93

3.2.5.1 Fabrication ...................... 94

3.2.5.2 Electrical characterization ............... 97

3.3 Single-Electron Transistors in Nanocrystalline Silicon ......... 101

3.3.1 Conduction in continuous nanocrystalline silicon films . . . 103

3.3.2 Nanocrystalline silicon nanowire SETs . . . . . . . . . 107

3.3.3 Point-contact nc-Si SET: Room temperature operation. .... . 111

3.3.4 'Grain-boundary' engineering . ............... 116

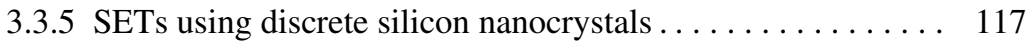

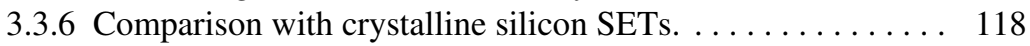

3.3.7 Electron coupling effects in nanocrystalline silicon . . . . . . 119

3.3.7.1 Electrostatic coupling effects ............. 121

3.3.7.2 Electron wavefunction coupling effects........ 122

3.4 Single-Electron Effects in Grown Si Nanowires and Nanochains. . 124

4. Single-Electron Memory 130

4.1 Introduction . . . . . . . . . . . . . . . . . . . . . . 130

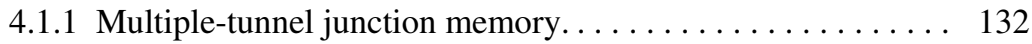

4.2 MTJ Memories in Silicon. . . . . . . . . . . . . . . . . . . 136

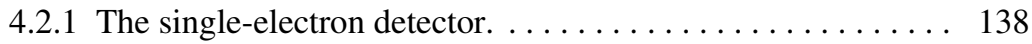

4.3 Single- and Few-Electron Memories with Floating Gates . . . . . . . 140

4.4 Large-scale Integrated Single-Electron Memory in Nanocrystalline Silicon ............................. 146

4.5 Few-Electron Memory with Integrated SET/MOSFET . . . . . . . . . 149

4.5.1 Silicon nanowire SETs for L-SEM application . . . . . . . 151

4.5.1.1 Nanowire SET in crystalline silicon. . . . . . . . . 153

4.5.1.2 Nanowire SET in polycrystalline silicon . . . . . . . 154

4.5.1.3 Potential for mass fabrication ............. 155

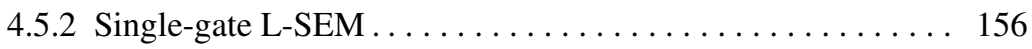

4.5.2.1 Single-gate L-SEM fabrication and characterization ...................... 159

4.5.3 Split-gate L-SEM . . . . . . . . . . . . . . . . . . . 163 
4.5.4 L-SEM $3 \times 3$ cell array. . . . . . . . . . . . . . 167

4.5.4.1 Memory cell selection . ............... 168

4.5.4.2 Temperature dependence of memory cell characteristics ................... 172

5. Few-Electron Transfer Devices 174

5.1 Introduction . . . . . . . . . . . . . . . . . . . . . . 174

5.2 Single-Electron Turnstiles and Pumps . . . . . . . . . . . . . . . . 175

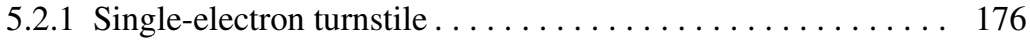

5.2 .2 Single-electron pump ..................... 179

5.2.3 Single-electron pump and turnstile using a semiconductor

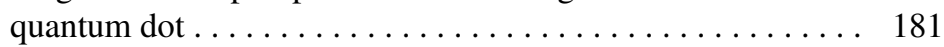

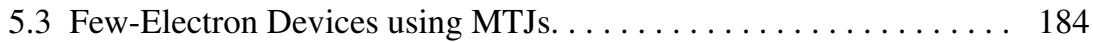

5.3.1 Operation of single r.f. signal MTJ electron pump. . . . . . 186

5.3.2 Single r.f. signal MTJ electron pumps in GaAs . . . . . . . . 191

5.3.3 Single r.f. signal MTJ electron pumps in silicon ......... 193

5.3.3.1 Device fabrication and experimental

Characteristics ....................... 195

5.3.4 MTJ electron pump with multi-phase r.f. signals. . . . . . . . 198

5.4 Single-Electron Transfer Devices in Silicon . . . . . . . . . . 200

5.4.1 Single-electron transfer using a CCD . . . . . . . . . . . . 201

5.4.2 SET/MOSFET single-electron pump and turnstile . . . . . 203

5.5 Metrological Applications . . . . . . . . . . . . . . . . . . 206

6. Single-Electron Logic Circuits 209

6.1 Introduction . . . . . . . . . . . . . . . . . . . . . . . 209

6.2 Voltage State Logic ....................... 210

6.2.1 SET inverter with resistive load ............... 211

6.2 .2 Complementary SET inverter . . . . . . . . . . . . . 213

6.2.3 Complementary SET NAND and NOR gates ......... 219

6.2.4 Programmable SET logic . . . . . . . . . . . . . . . . . 222

6.2.5 Logic using SETs with multiple input terminals . . . . . . . 225

6.2.6 Effect of offset charge ..................... 227

6.3 Charge State Logic . . . . . . . . . . . . . . . . . . . . . . 228

6.3.1 Binary decision diagram logic ................ 229

6.3.1.1 Binary decision diagram logic:

Basic logic gates ................... 231

6.3.1.2 Implementation of BDD logic circuits in GaAs. . . . 237

6.3.2 Implementation of BDD logic circuits in silicon. ........ 245

6.3.2.1 Two-way BDD switch using silicon nanowire SETs. . . . . . . . . . . . . . . . . . 246 
6.3.2.2 Extension to a 'universal' three-way switch ...... 250

6.4 Quantum Cellular Automaton Circuits. ............... 252

6.5 Single-Electron Parametron . . . . . . . . . . . . . . . 258

$\begin{array}{ll}\text { Bibliography } & 261\end{array}$

$\begin{array}{ll}\text { Index } & 281\end{array}$ 